\title{
Macrophytes of the lowland wetlands in Morang district, Nepal
}

\author{
Umesh Koirala and Sasinath Jha \\ Department of Botany, Post Graduate Campus, Tribhuvan University, Biratnagar, Nepal \\ E-mail:umesh6977@hotmail.com
}

\begin{abstract}
In total, 149 species of macrophytes (angiosperms 138, pteridophytes 7, bryophytes 2 , algae 2) were recorded among which 117 species were emergent, 12 floating-leafed, 14 submerged and 6 free-floating on the basis of life form. In general, 68\% species had flowering-fruiting during the rainy season, $17 \%$ in winter and $15 \%$ in the summer season. Many of the plant species were observed to play important role in meeting day to day requirements of the rural people.
\end{abstract}

Key words: Ethnobotany, life form, macrophytes, reproductive phase, wetlands.

\section{Introduction}

Known as 'Simsar' in Nepal, wetlands are those areas which lie between the land and deepwater and remain waterlogged or submerged under water, seasonally or throughout the year. Generally, the land is so muddy that one cannot easily walk over it, and water is so deep that one can neither swim nor get drowned. River floodplains, shallow margins of lakes and reservoirs, depressions/ditches, man-made ponds, islands in rivers, marshes, swamps and deepwater paddy fields are typical examples of freshwater wetlands found in Nepal (Jha, 2008). Wetland macrophytes are grouped into five categories: obligate wetland plants (OBL) that occur almost always in wetlands under natural conditions; facultative wetland plants (FACW) that occur usually in wetlands, but also occur in nonwetlands; facultative plants (FAC) with a similar likelihood of occurring in both wetlands and nonwetlands; facultative upland plants (FACU) that occur sometimes in wetlands, but occur more often in nonwetlands; and obligate upland plants (UPL) that occur rarely in wetlands, but occur almost always in nonwetlands under natural condition (Mitsch \& Gosselink, 2000). This paper enumerates macrophytes of OBL, FACW and FAC categories that occur in the lowland wetlands (a part of the Indo-Gangetic floodplain) of Morang district along with their life form, season for flowering-fruiting/spore formation, locality, habitat and human use (s) value.

\section{Materials and Methods}

All types of wetlands occurring at five locations (Biratnagar, Indrapur, Baghjhoda, Betana and Madhumalla) in the lowland region $\left(26^{\circ} 20^{\prime}\right.$ to $26^{\circ} 45^{\prime} \mathrm{N}$ and $87^{\circ} 15^{\prime}$ to $87^{\circ}$ $30^{\prime} \mathrm{E}$, altitudes 72 to $300 \mathrm{~m} \mathrm{msl}$ ) of Morang district were surveyed for macrophytes during January, 2009 to December, 2010. Climate of the lowland region of Morang district is tropical and monsoonic with three seasons in a year, viz., rainy (mid-June to October), winter (November to February), and summer (March to mid- June). The maximum and minimum annual temperature is $30.6^{\circ} \mathrm{C}$ and $14.2^{\circ} \mathrm{C}$, respectively. July 
is the hottest month while January is the coldest month. The average annual rainfall is about $1312 \mathrm{~mm}$, of which $85 \%$ is received during the rainy season.

Plant materials and field data were gathered by making regular visits at monthly intervals to the selected locations. Generally, four to five specimens of each species were collected after recording field data such as life from, habitat, and phenology. The collected specimens were properly dried and treated with $0.1 \%$ solution of mercuric chloride for 1 to $2 \mathrm{~min}$ before mounting them on the herbarium sheet (Jha and Jha, 2000). The herbarium specimens were identified with the help of standard literature (Banerji, 1972; Hooker, 1872-1897; Iwatsuki, 1988; Cook, 1996; Siwakoti \& Varma, 1999), and all the identifications were confirmed further by cross-checking with the authentic specimens at the National Herbarium and Plant Laboratories, Godavari (KATH). Scientific names of the specimens provided by Hara et al. (1978, 1979, 1982), Iwatsuki (1988), Koba et al. (1994), Cook (1996) and Press et al. (2000) were adopted for nomenclature.

Information on use(s) of the macrophytes were collected either through direct consultation with local practitioners or through consulting standard literature (CSIR, 1992; Manandhar, 1998).

\section{Results and Discussion}

In total 149 species of macrophytes were recorded in the present study among which angiosperms were represented by 45 families (dicots 28, monocots 17), 103 genera (dicots 48, monocots 55), and 138 species (dicots 60, monocots 78); pteridophytes by 6 families, 6 genera and 7 species; bryophytes by 2 families, 2 genera and 2 species, and algae by only one family, 2 genera and 2 species (Table 1). The ten dominant families of angiosperms occurred in the order: Poaceae (26 species) > Cyperaceae (21) $>$ Asteraceae (8) > Scrophulariaceae (5) > Acanthaceae (4), Araceae (4), Commelinaceae (4), Hydrocharitaceae (4), Polygonaceae (4), and Potamogetonaceae (4). The dicot genera with two or more species were Alternanthera, Ammannia, Hygrophila, Ipomoea, Limnopila, Lindernia, Ludwigia, Nymphoides, Persicaria, and Rorippa. The genera represented by two or more species among the monocots were Cyperus, Echinochloa, Eragrostis, Fimbristylis, Kyllinga, Leptochloa, Monochoria, Panicum, Paspalum, Potamogeton, Sacciolepis, Sagittaria, and Schoenoplectus. Only one genus (Equisetum) was represented by two species among the pteridophytes.

The invasive alien species (IAS) occurring in the wetlands were Alternanthera philoxeroides, Eichhornia crassipes, Ipomoea carnea and Mikania micrantha.

Phenological information can be used to study animal-plant interactions which affect pollination, seed dispersal, and fruit/seed predation. These interactions are important for plant reproduction and reciprocally for food for the animals involved (Jha, 2005). Observations made on the recorded macrophytes revealed that $68 \%$ species attained reproductive phase in rainy, $17 \%$ in winter, and $15 \%$ in the summer season.

Analysis of life form indicated that 117 species of the recorded macrophytes were emergents, 12 floating-leafed, 14 submerged, and 6 free-floating. Both the freefloating and floating-leafed species provide a nesting habitat for birds such as the pheasant-tailed Jacana, bronze-winged Jacana, and purple Moorhen (Sankhala, 1990). The submerged macrophytes provide food for fish and a habitat for large numbers of 
Table 1. Wetland macrophytes and their uses (Life forms: $E=$ emergent; FL= floating -leafed; FF= free-floating; $\mathrm{S}=$ submerged). $\mathrm{LF}=\mathrm{Life}$ form, $\mathrm{SFFSF}=\mathrm{Season}$ for floweringfruiting/spore formation

\begin{tabular}{|c|c|c|c|c|c|c|c|}
\hline Family & Plant species & $\mathbf{L F}$ & SFFSF & Locality & Habitat & Parts used & Uses \\
\hline \multicolumn{8}{|l|}{ A. Dicotyledons } \\
\hline \multirow[t]{3}{*}{ 1. Acathaceae } & Hygrophila auriculata (Schumach.) Heine & $\mathrm{E}$ & Oct.-Apr. & Biratnagar-4 & Edge of ditches & - & - \\
\hline & H. difformis (L.f.) Blume & $\mathrm{E}$ & Aug.-Dec. & Betana & Edge of ditches, marshes & - & - \\
\hline & $\begin{array}{l}\text { Phlogacanthus pubinervius } \mathrm{T} \text {. } \\
\text { Anderson }\end{array}$ & $\mathrm{E}$ & Feb.-Mar. & Betana & Marshes & - & Pot-herb \\
\hline \multirow[t]{3}{*}{ 2. Amaranthaceae } & Alternanthera paronychioides St. Hil. & $\mathrm{E}$ & Aug.-Dec. & Biratnagar-4 & Moist soil & Shoot & Green manure/compost \\
\hline & A. philoxeroides (Mart.) Griseb & $\mathrm{E}$ & Mar.-Jun. & Biratnagar-10 & Muddy ponds, canals \& ditches & Whole plants & Pot-herb, medicinal, IAS \\
\hline & Gomphrena celosioides Mart. & $\mathrm{E}$ & All seasons & Biratnagar-18 & Moist soil & - & Feed for livestock. \\
\hline \multirow[t]{3}{*}{ 3. Apiaceae } & Centella asiatica (L.) Urban & $\mathrm{E}$ & All seasons & Biratnagar-18 & Moist soil & Whole plant & $\begin{array}{l}\text { Used as an diuretic, decoction useful in } \\
\text { leprosy \& nervous disorder. }\end{array}$ \\
\hline & Hydrocotyle sibthorpioides Lam. & $\mathrm{E}$ & Dec.-Jan. & Madhumalla & Moist soil & - & - \\
\hline & Oenanthe javanica (Blume) DC. & $\mathrm{E}$ & May.-Jul. & Biratnagar-18 & Marshes & Shoot & Pot-herb. \\
\hline 4. Asclepiadaceae & Oxystelma esculentum (L.f.) Sm. & $\mathrm{E}$ & Aug.-Nov. & Biratnagar-10 & Moist soil & Root & Medicinal \\
\hline \multirow[t]{7}{*}{ 5. Asteraceae } & Caesulia axillaris Roxb. & $\mathrm{E}$ & Sep.-Feb. & Biratnagar-6 & Wet paddy field & Shoot & Fodder, Medicinal \\
\hline & Eclipta prostrata $(\mathrm{L}.) \mathrm{L}$ & $\mathrm{E}$ & All seasons & Biratnagar-15 & Edge of water course, moist soil & Shoot & Medicinal, religious \\
\hline & Gnaphalium affine D. Don. & $\mathrm{E}$ & Feb.-Oct. & Biratnagar-18 & Moist soil & - & - \\
\hline & Grangea maderaspatana (L.) Poir. & $\mathrm{E}$ & All seasons & Biratnagar-15 & Moist soil & Whole plant & Green manure/compost, Medicinal \\
\hline & Mikania micrantha Kunth & $\mathrm{E}$ & Nov.-Feb. & Biratnagar-18 & Moist soil, ditches \& ponds & - & IAS. \\
\hline & Sphaeranthus indicus L. & $\mathrm{E}$ & Feb.-Apr. & Biratnagar-18 & Wet paddy fields & Shoot & Insect repellent \\
\hline & Spilanthes iabadicensis A.H. Moore & $\mathrm{E}$ & All seasons & Biratnagar-4 & Marshes, edge of water bodies & - & - \\
\hline 6. Boraginaceae & Heliotropium indicum L. & $\mathrm{E}$ & Oct.- Dec. & Biratnagar-15 & Dry ditches & - & - \\
\hline \multirow[t]{2}{*}{ 7. Brassicaceae } & Rorippa benghalensis (DC.) H. Hara & $\mathrm{E}$ & Apr.-Jun. & Biratnagar-15 & Moist soil & Shoot & Medicinal \\
\hline & R. nasturtium-aquaticum (L.) Hayek & $\mathrm{E}$ & Apr.-Jun. & Biratnagar-18 & Marshes & Shoot & Pot-herb, Medicinal \\
\hline 8. Cabombaceae & Cabomba aquatica Aublet & $\mathrm{S}$ & Jun.-Oct. & Betana & Silty ponds & Whole plant & Fodder \\
\hline 9. Ceratophyllaceae & Ceratophyllum demersum $\mathrm{L}$. & $\mathrm{S}$ & All seasons & Biratnagar -18 & Silty ponds, canals & Leaves & $\begin{array}{l}\text { In scorpion sting, antipyretic, cooling } \\
\text { effect, hyper accumulator of heavy metals }\end{array}$ \\
\hline 10. Convulvulaceae & Impomea aquatica Forrsk. & FL & Nov.- Jan. & Biratnagar-4 & Ponds, ditches & Leaf & Pot-herb, Juice emetic, \& purgative \\
\hline
\end{tabular}




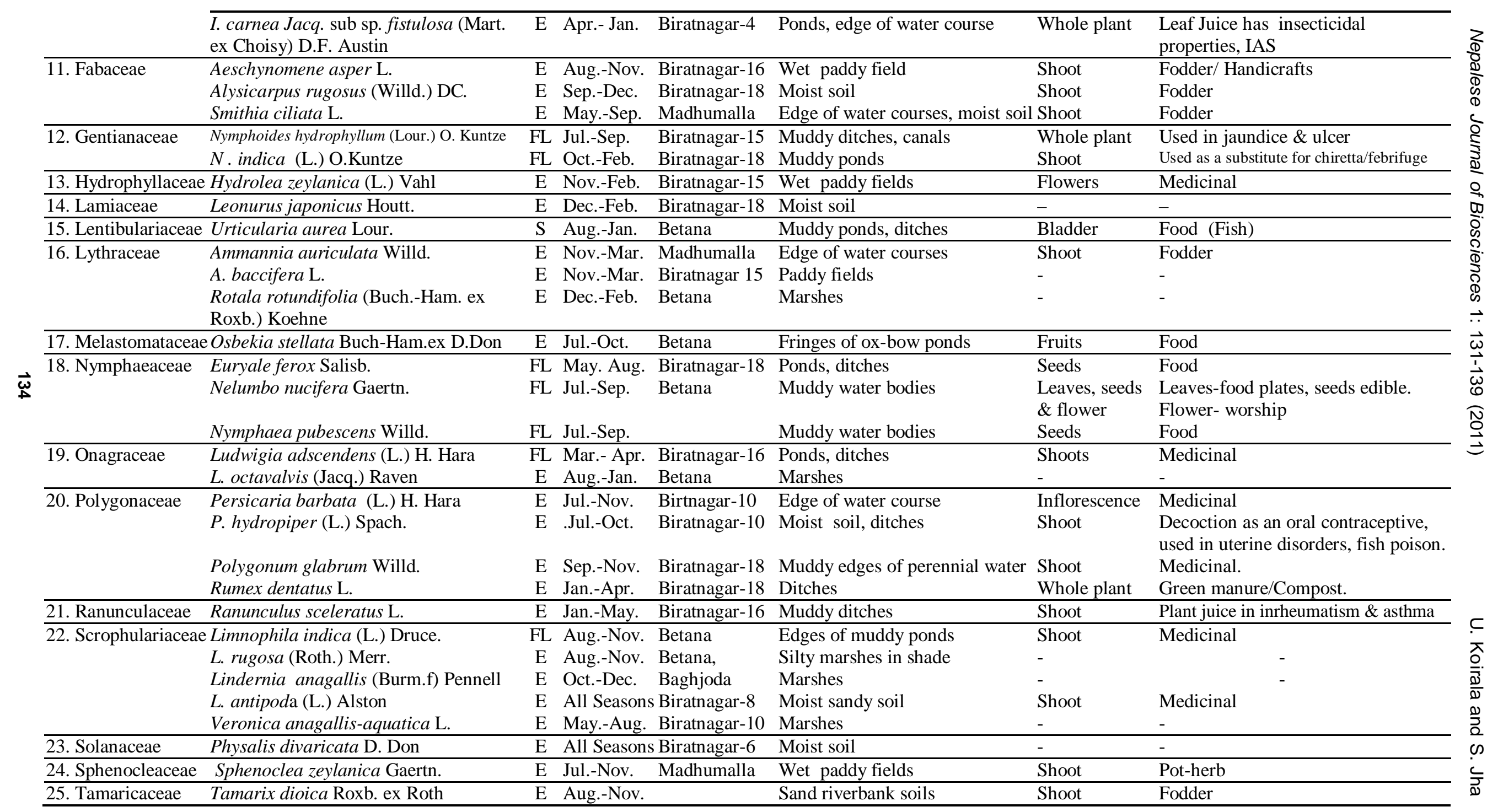




\begin{tabular}{|c|c|c|c|c|c|c|c|}
\hline 26. Trapaceae & Trapa qudrispinosa Roxb. & FL & Mar.-Jul. & Biratnagar-18 & Cultivated, ponds, ditches & Fruits & Fruit edible \\
\hline 27. Urticaceae & Pouzolzia zeylanica (L.) J. Bennett \& Br. & $\mathrm{E}$ & Sep.-Nov. & Biratnagar-18 & Marshes & Leaf & Medicinal \\
\hline 28. Verbinaceae & Lippia nodiflora (L.) Rich. & $\mathrm{E}$ & All Seasons & Biratnagar-18 & Shady moist places & Whole plant & Medicinal \\
\hline \multicolumn{8}{|l|}{ B. Monocotyledons } \\
\hline 29. Alismataceae & $\begin{array}{l}\text { Sagittaria guyanensis Kunth } \\
\text { S. trifolia L. }\end{array}$ & $\begin{array}{c}\text { FL } \\
\mathrm{E}\end{array}$ & $\begin{array}{l}\text { Sep.-Nov } \\
\text { Jun.-Sep. }\end{array}$ & $\begin{array}{l}\text { Biratnagar-4 } \\
\text { Betana }\end{array}$ & $\begin{array}{l}\text { Ditches, paddy fields } \\
\text { Marshes }\end{array}$ & $\begin{array}{l}\text { Rhizome } \\
\text { Young leaves }\end{array}$ & $\begin{array}{l}\text { Pot-herb } \\
\text { Pot--herb }\end{array}$ \\
\hline 30. Amaryllidaceae & Crinum asiaticum Roxb. & $\mathrm{E}$ & Jul.-Aug. & Biratnagar-4 & Edge of ditches & - & - \\
\hline 31. Aponogetonaceae & Aponogeton appendiculatus $\mathrm{H}$. Bruggen & FL & Sep.-Feb. & Betana & Sandy bottom of running water & Tubers & Food \\
\hline 32. Araceae & $\begin{array}{l}\text { Acorus calamus } \mathrm{L} \text {. } \\
\text { Colocasia esculenta }(\mathrm{L} .) \text { Schott } \\
\text { Lasia spinosa }(\mathrm{L} .) \text { Thwaites } \\
\text { Pistia stratiotes } \mathrm{L} .\end{array}$ & $\begin{array}{c}\mathrm{E} \\
\mathrm{E} \\
\mathrm{E} \\
\mathrm{FF}\end{array}$ & $\begin{array}{l}\text { Mar.-Jun. } \\
\text { Aug,.-Sep. } \\
\text { Sep.-Nov. } \\
\text { Aug.-Feb. }\end{array}$ & $\begin{array}{l}\text { Biratnagar-1 } \\
\text { Biratnagar-10 } \\
\text { Betana } \\
\text { Biratnagar-15 }\end{array}$ & $\begin{array}{l}\text { Marshes } \\
\text { Muddy ditches, canals } \\
\text { Oxbow pond/silty-marshes } \\
\text { Ponds, ditches }\end{array}$ & $\begin{array}{l}\text { Rhizome } \\
\text { Whole plant } \\
\text { Shoot } \\
\text { Whole plant }\end{array}$ & $\begin{array}{l}\text { Medicinal/Insect repellents } \\
\text { Vegetable /Fodder } \\
\text { Vegetable } \\
\text { Plant as manure, juice in earache, ashes in } \\
\text { ringworm. Leaves eczema, leprosy, ulcer }\end{array}$ \\
\hline 33. Butomaceae & Butomopsis latifolia (D.Don) Kunth & $\mathrm{E}$ & Sep.-Nov. & Madhumalla & Wet paddy fields & - & - \\
\hline 34. Cannaceae & Canna indica $\mathrm{L}$. & $\mathrm{E}$ & All Seasons & Biratnagar-15 & Muddy ditches & - & - \\
\hline \multirow[t]{4}{*}{ 35. Commelinaceae } & $\begin{array}{l}\text { Amischophacelus axillaris (L.) Rao \& } \\
\text { Kammathy }\end{array}$ & $\mathrm{E}$ & Aug.-Nov. & Biratnagar-18 & Moist places & - & - \\
\hline & Commelina benghalensis L. & $\mathrm{E}$ & Jul.-Jan. & Biratnagar-18 & Edges of water, marshes \& ditches & Shoot & Useful in leprosy \\
\hline & Floscopa sc\&ense Lour. & $\mathrm{E}$ & Sep.-Dec. & Betana & Edges of water courses & Shoot & Fodder \\
\hline & Murdania nudiflora L.Brenan & $\mathrm{E}$ & Aug.-Oct. & Biratnagar-18 & Paddy fields & Shoot & Fodder \\
\hline \multirow[t]{15}{*}{ 36. Cyperaceae } & Carex nubigena D.Don & $\mathrm{E}$ & Aug.-Nov. & Betana & Marshes & Shoot & Fodder \\
\hline & Cyperus amabilis Vahl & $\mathrm{E}$ & Aug.-Oct. & Madhumalla & Wet paddy field & - & - \\
\hline & C. compressus $\mathrm{L}$. & $\mathrm{E}$ & Sep-Oct. & Biratnagar-10 & Marshes & Shoot & Fodder \\
\hline & C. corymbosus Rottb. & $\mathrm{E}$ & Sep.-Nov. & Madhumalla & Marshes & Shoot & H\&icrafts \\
\hline & C. difformis $\mathrm{L}$. & $\mathrm{E}$ & Aug.- Nov. & Biratnagar-18 & Marshes, ditches & - & - \\
\hline & C. esculentus $\mathrm{L}$. & $\mathrm{E}$ & Aug.- Nov & Biratnagar-18 & Marshes, paddy fields & Shoot & H\&icrafts \\
\hline & C. iria $\mathrm{L}$. & $\mathrm{E}$ & Sep.-Nov. & Biratnagar-4 & Paddy fields & Whole plant & Medicinal \\
\hline & C. natus Vahl & $\mathrm{E}$ & Aug.-Oct. & Betana & Muddy edge of ponds & Shoot & Fodder \\
\hline & C. pilosus Vahl & $\mathrm{E}$ & Sep.- Nov & Biratnagar-7 & Swamps & - & - \\
\hline & C. platystylis $\mathrm{R} . \mathrm{Br}$. & $\mathrm{E}$ & Sep.- Nov & Betana & Sandy moist soil & - & - \\
\hline & Eleocharis atropurpurea (Retz.) Presl & $\mathrm{E}$ & Nov.-Dec. & Biratnagar-8 & Rice field & Shoot & Fodder \\
\hline & Fimbristylis dichotoma (L.) Vahl & $\mathrm{E}$ & Jun.-Nov. & Biratnagar-18 & Edge of ponds & Shoot & Fodder \\
\hline & F. littoralis Gaud. & $\mathrm{E}$ & Jun.-Nov. & Biratnagar-18 & Moist paddy fields & Shoot & Fodder \\
\hline & F. schoenoides (Retz.) Vahl & $\mathrm{E}$ & Set.-Nov & Biratnagar-18 & Wet paddy fields & Shoot & Fodder \\
\hline & Kyllinga brevifoia Rottb. & $\mathrm{E}$ & Jul.-Nov. & Biratnagar-10 & Moist soil & Shoot & Fodder \\
\hline
\end{tabular}




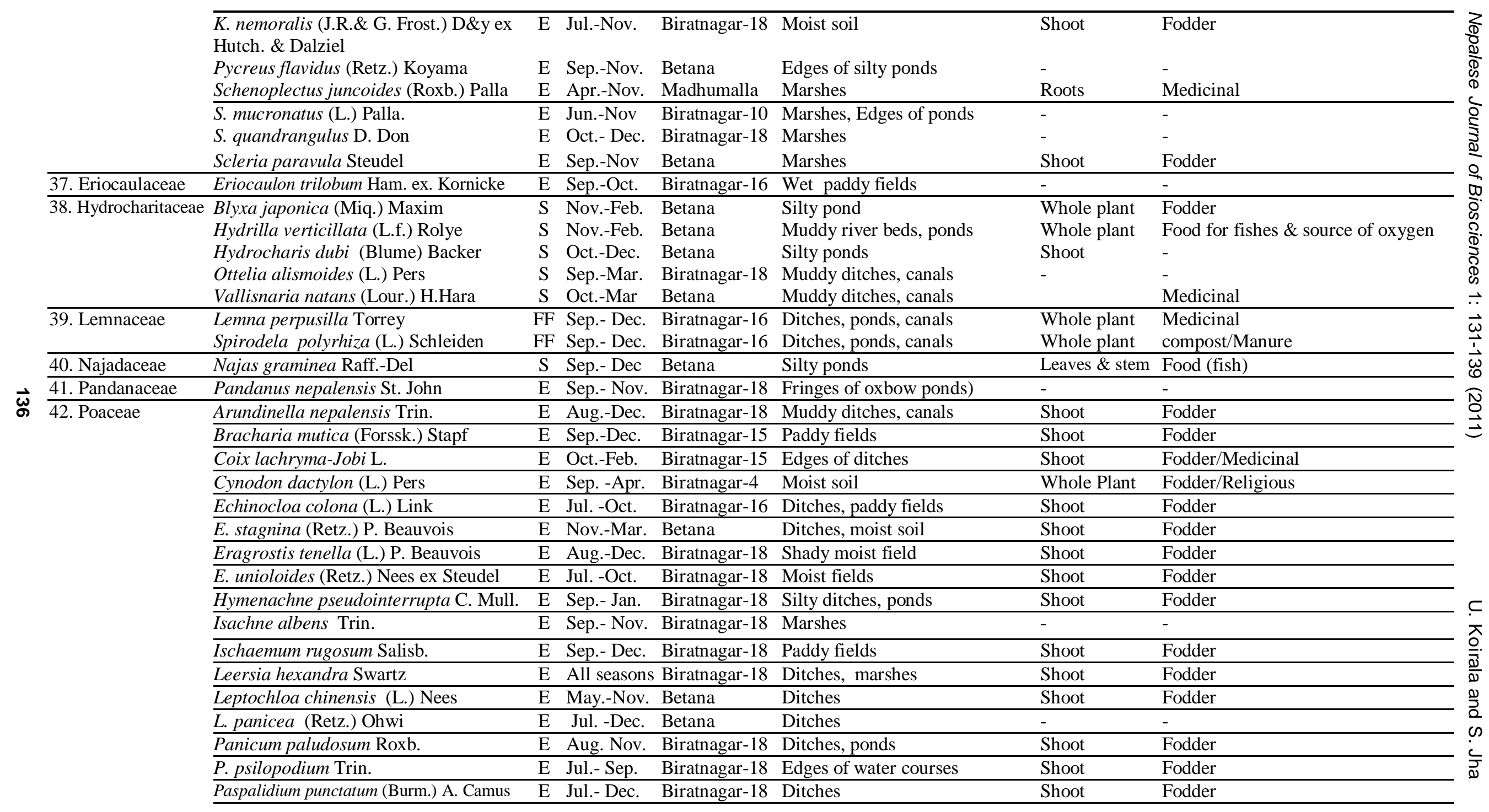




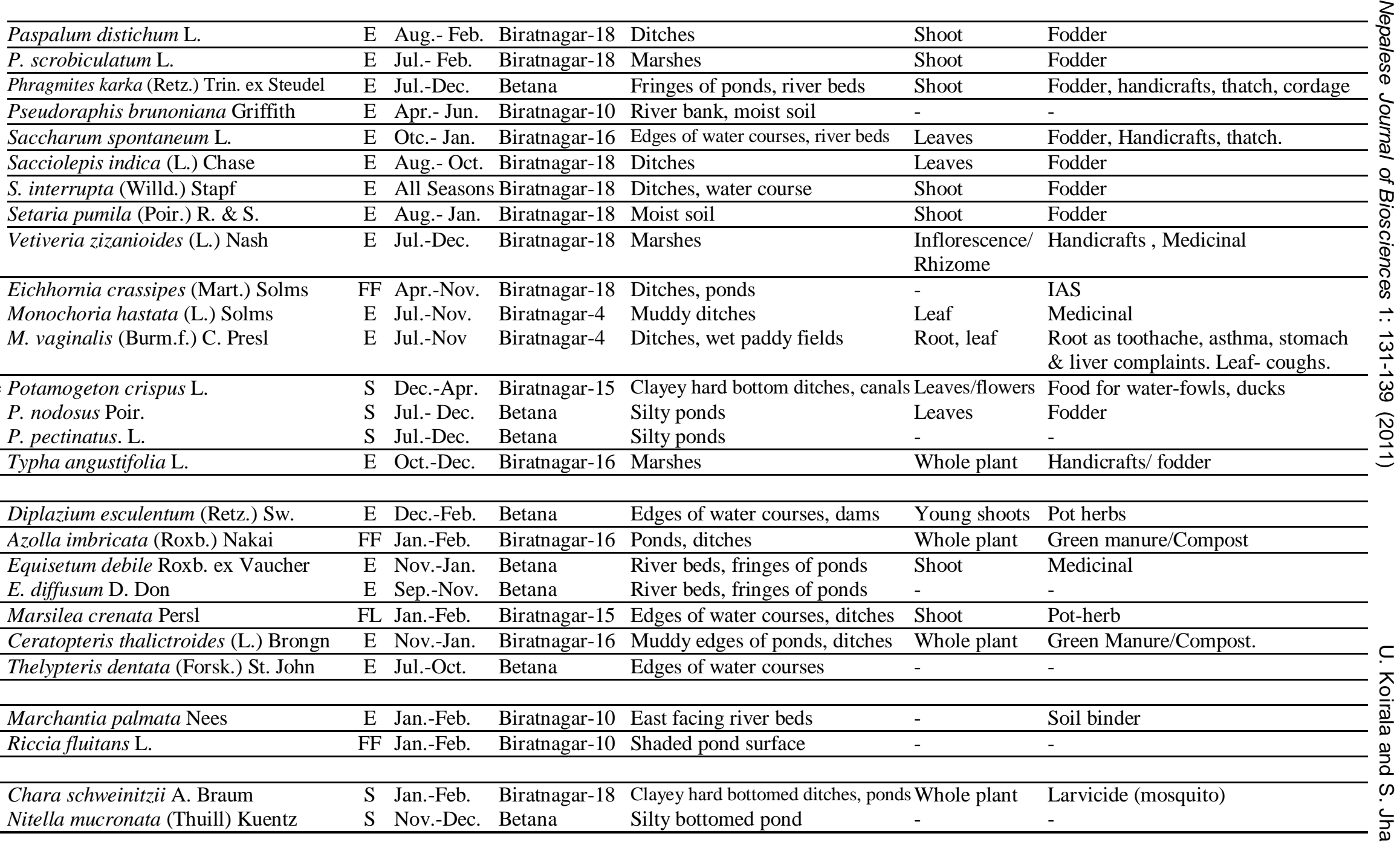


invertebrates. The leaves and shoots of emergent macrophytes provide food for grasshoppers and habitats for several bird species.

The rural people were observed to use most of the macrophytes listed in Table 1 as food, fodder, medicine, green manure/compost, insect repellent, etc. Macrophytes recorded as wild food plants were Alternanthera philoxeroides (pot-herb), Diplazium esculentum (pot-herb), Euryale ferox (seeds), Ipomoea aquatica (pot-herb), Nelumbo nucifera (seeds), Rorippa nasturtium-aquaticum (pot-herb), Trapa quadrispinosa (fruits) etc. Notable species used as fodder were Alysicarpus rugosus, Arundinella nepalensis, Blyxa japonica, Brachiaria mutica, Cabomba aquatica, Carex nubigena, Coix lachryma-jobi, Colocasia esculenta, Cynodon dactylon, Echinochloa colona, Panicum psilopodium, Paspalum distichum, P. scrobiculatum, Sacciolepis indica and Vetiverria zizanioides. Macrophytes used for treating various diseases and ailments were Acorus calamus, Centella asiatica, Ceratophyllum demersum, Commelina benghalensis, Eclipta prostrata, Equisetum debile, Hydrolea zeylanica, Lippia nodiflora, Ludwigia adscendens, Monochoria hastata, Ottelia alisomoides, Oxystelma esculentum, Persicaria barbata, Ranunculus sceleratus, Rorippa benghalensis, Vetiveria zizanioides, etc. Macrophytes used in traditional crafts were Aeschynomene asper, Cyperus corymbosus, C. esculentus, Typha angustifolia and Vetiveria zizanioides; whereas plants used as green manure/compost were Azolla imbricata, Eichhornia crassipes, Pistia stratiotes, Rumex dentatus and Spirodela polyrhiza.

Since time immemorial the rural people (particularly fisher-folk and other ethnic communities) have depended on wetlands for fishing, deepwater paddy cultivation, wild food/vegetables, medicinal herbs, reeds and mud for house construction, and specific plant species for traditional crafts. Hence, establishment of small scale cottage industries for the utilization of the macrophytes for various purposes will certainly provide adequate employment opportunities for the wetlanders.

\section{Acknowledgements}

One of the authors (UK) is grateful to the Tribhuvan University for the Ph.D. study leave and to the University Grants Commission, Nepal for the research fellowship.

\section{References}

Banerji, M.L. 1972. A collection of ferns from eastern Nepal. Candollea 27(2): 268-281.

Cook, C.D.K. 1996. Aquatic and wetland plants of India. Oxford University Press, Delhi.

CSIR (Council of Scientific and Industrial Research) 1992. The wealth of India: Raw materials. vols. I-IX. Publication and Information Directorate, CSIR, New Delhi.

Hara, H. \& L.H.J Williams (eds.). 1979. An enumeration of the flowering plants of Nepal. vol. 2. British Museum (Natural History), London.

Hara, H., A.O. Chater \& L.H.J. Williams (eds.). 1982. An enumeration of the flowering plants of Nepal. vol. 3. British Museum (Natural History), London.

Hara, H., W.T. Stearn \& L.H.J. Williams (eds.). 1978. An enumeration of the flowering plants of Nepal. vol. 1. British Museum (Natural History), London.

Hooker, J.D. 1872-1897. The flora of British India. 7 vols. L. Reeve, London.

Iwatsuki, K. 1988. An enumeration of the pteridophytes of Nepal. In: The Himalayan Plants. vol. 1. (Eds. H. Ohba \& S.B. Malla). University of Tokyo, Tokyo. pp. 231339.

Jha, S. \& P.K. Jha. 2000. Contribution to the flora of Morang district and adjoining areas of Nepal. Lidia (A Norwegian Journal of Botany) 5(1-2): 25-64. 
Jha, S. 2005. Comparative analysis of the flora of Morang district and adjoining areas of Nepal. Our Nature 3: 63-68.

Jha, S. 2008. Status and conservation of lowland Terai wetlands in Nepal. Our Nature 6: 67-77.

Koba, H., S. Akiyama, Y. Endo \& H. Ohba. 1994. Name list of the flowering plants and gymnosperms of Nepal. University of Tokyo, Tokyo.

Manandhar, N.P. 1998. Native phytotherapy among the Route tribes of Dadeldhura district. Journal of Ethnopharmacology 60(3): 199-206.

Mitsch, W.J. \& J.G. Gosselink. 2000. Wetlands. $3^{\text {rd }}$ ed. John Wiley \& Sons, Inc., New York.

Press, J.R., K.K. Shrestha \& D.A. Sutton. 2000. Annotated checklist of the flowering plants of Nepal. The Natural History Museum, London.

Sankhala, K. 1990. Gardens of Gods: The waterbird sanctuary at Bharatpur. Vikas Publishing House, New Dehli.

Siwakoti, M. \& S.K. Varma. 1999. Plant diversity of eastern Nepal. Bishen Singh Mahendra Pal Singh, Dehra Dun. 